\title{
On the Uniqueness of Simultaneous Rational Function Reconstruction
}

\author{
Eleonora Guerrini, Romain Lebreton, Ilaria Zappatore \\ guerrini,lebreton,zappatore@lirmm.fr \\ LIRMM, Université de Montpellier, CNRS \\ Montpellier, France
}

\begin{abstract}
This paper focuses on the problem of reconstructing a vector of rational functions given some evaluations, or more generally given their remainders modulo different polynomials. The special case of rational functions sharing the same denominator, a.k.a. Simultaneous Rational Function Reconstruction (SRFR), has many applications from linear system solving to coding theory, provided that SRFR has a unique solution. The number of unknowns in SRFR is smaller than for a general vector of rational function. This allows to reduce the number of evaluation points needed to guarantee the existence of a solution, but we may lose its uniqueness. In this work, we prove that uniqueness is guaranteed for a generic instance.
\end{abstract}

\section{INTRODUCTION}

The Vector rational function reconstruction (VRFR) is the problem of finding all rational functions $\boldsymbol{v} / \boldsymbol{d}=\left(v_{1} / d_{1}, \ldots, v_{n} / d_{n}\right)$ which satisfy some degree constraints, given a certain number of their evaluations $(\boldsymbol{v} / \boldsymbol{d})\left(\alpha_{j}\right)=\omega_{j}$. We consider a generalized version of this problem, where we suppose to know the images modulo different polynomials $a_{1}, \ldots, a_{n}$, i.e. $u_{i}=v_{i} / d \bmod a_{i}$ for $1 \leq i \leq n$. The Simultaneous Rational Function Reconstruction (SRFR) problem is a particular case of the vector rational function reconstruction where the rational functions $\boldsymbol{v} / d=\left(v_{1} / d, \ldots, v_{n} / d\right)$ share the same denominator (see Section 2.1). We can apply the SRFR in different problems: from the decoding of classic and interleaved Reed-Solomon codes to the polynomial linear system solving. As in the classic rational function reconstruction we focus on the homogeneous linear system related to our equations in its weaker form, i.e. $\boldsymbol{v}-d \boldsymbol{u} \equiv 0 \bmod \boldsymbol{a}$. If the number of equations is equal to the number of unknowns minus one then there always exists a non-trivial solution. From now on, we will assume to be in this case. Note that the common denominator constraint of SRFR implies less unknowns than general VRFR, so less equations. This has a direct impact on the complexity of its applications. However, the uniqueness in not anymore guarantied as shown in Counterexample 2.2. Having a unique solution is fundamental for decoding algorithms or Evaluation-Interpolation methods (like for instance in linear system solving). This paper focuses on the conditions that guarantee the uniqueness of solutions of the SRFR.

Previous works show that in the application of SRFR for polynomial linear system solving, the uniqueness is ensured under some specific degree conditions [OS07]. We have reasons to believe that we can generalize this result: we conjecture that for almost all $(\boldsymbol{v}, d)$ the SRFR problem admits a unique solution (see Conjecture 2.5).

We can learn more about the conditions of uniqueness from the results coming from error correcting codes. Interleaved Reed
Solomon Codes (IRS) can be seen as the evaluation of a vector of polynomials $\boldsymbol{v}$. The problem of decoding IRS codes consists in the reconstruction of the vector of polynomials $v$ by its evaluations, some possibly erroneous. A classic approach to decode IRS codes is the application of the SRFR for instances $\boldsymbol{u}=\boldsymbol{v}+\boldsymbol{e}$ where $\boldsymbol{e}$ are the errors. Results from coding theory show that for all $v$ and almost all errors $\boldsymbol{e}$, we get the uniqueness of SRFR for the corresponding instance $\boldsymbol{u}$ (provided that there are not too many errors) [BKY03, BMS04, SSB09]. There is a natural generalization of SRFR when errors occur (SRFRwE, see Section 2.2), which can be seen as fractional generalization of IRS [GLZ19, GLZ20]. We conjecture that we can decode almost all codeword $(\boldsymbol{v} / d)$ and almost all errors $\boldsymbol{e}$ of this fractional code (Conjecture 2.9). In this paper we present a result which is a step towards this conjecture. We prove that uniqueness is guaranteed for a generic instance $\boldsymbol{u}$ of SRFR, (Theorem 5.2). Our result is valid not only given evaluations, but also in the general context of any moduli $a$.

Our approach to prove Theorem 5.2 is to study the degrees of a relation module. Solutions of SRFR are related to generators of a row reduced basis of this $\mathbb{K}[x]$-module which have a negative shifted-row degree. Shifts are necessary to integrate degree constraints. We show that for generic instances, there is only one generator with negative row degree, hence uniqueness of the SRFR solution.

Previous works studied generic degrees of different but related modules: e.g. for the module of generating polynomials of a scalar matrix sequence [Vil97], for the kernel module of a polynomial matrix and specific matrix dimensions [JV05]. Both cases does not consider any shift. The generic degrees also appear in dimensions of blocks in a shifted Hessenberg form. However, the link with the degree of a module is unclear and no shift is discussed (shifted Hessenberg is not related to our shift) [PS07]. We prove our result for any shift and any matrix dimension by adapting some of their techniques, and by proving that they apply to the specific relation modules related to SRFR.

In Section 2 we introduce the motivations of our work, started from the classic SRFR to the extended version with errors. We also show their respective applications in polynomial linear system solving and in error correcting algorithms. In Section 3, we define the algebraic tools that we will use to prove our technical results of the Section 4. In Section 5 we explain how these results are linked to the uniqueness of the solution of the SRFR and we finally prove the Theorem 5.2 about the generic uniqueness. 


\section{MOTIVATIONS}

\subsection{Rational Function Reconstruction}

In this section we recall standard definitions and we state our problem, starting from rational function reconstruction and its application to linear algebra. Let $\mathbb{K}$ be a field, $a, u \in \mathbb{K}[x]$ with $\operatorname{deg}(u)<$ $\operatorname{deg}(a)$. The Rational Function Reconstruction (shortly RFR) is the problem of reconstructing a rational function $v / d \in \mathbb{K}(x)$ such that

$$
\operatorname{gcd}(d, a)=1, \frac{v}{d} \equiv u \bmod a, \operatorname{deg}(v)<N, \operatorname{deg}(d)<D .
$$

We focus on the weaker equation:

$$
v \equiv d u \bmod a, \operatorname{deg}(v)<N, \operatorname{deg}(d)<D .
$$

The RFR problem generalizes many problems including the Padé approximation if $a=x^{f}$ and the Cauchy interpolation if $a=\prod_{i=1}^{f}(x-$ $\alpha_{i}$ ), where the $\alpha_{i}$ are pairwise distinct elements of the field $\mathbb{K}$. The homogeneous linear system related to the Equation (2) has $\operatorname{deg}(a)$ equations and $N+D$ unknowns. If $\operatorname{deg}(a)=N+D-1$, the dimension of the solution space of Eq. (2) is at least 1 and it always admits a non-trivial solution. Moreover, such a solution is unique in the sense that all solutions are polynomial multiples of a unique one, $\left(v_{\min }, d_{\min }\right)$ (see e.g. [GG13, Theorem 5.16]). On the other hand, Equation (1) does not always have a solution, but when a solution exists, it is unique. Indeed, it is $v_{\min } / d_{\min }$ and we can reconstruct it by the Extended Euclidean Algorithm (EEA). Throughout this paper, we will focus on Equation (2).

The RFR can be naturally extended to the vector case as follows. Let $a_{1}, \ldots, a_{n} \in \mathbb{K}[x]$ with degrees $f_{i}=\operatorname{deg}\left(a_{i}\right)$ and $\boldsymbol{u}=$ $\left(u_{1}, \ldots, u_{n}\right) \in \mathbb{K}[x]^{n}$ where $\operatorname{deg}\left(u_{i}\right)<f_{i}$. Let $0<N_{i}, D_{i}<f_{i}$. The Vector Rational Function Reconstruction (VRFR) is the problem of reconstructing $\left(v_{i}, d_{i}\right)$ for $1 \leq i \leq n$ such that $v_{i} \equiv d_{i} u_{i} \bmod$ $a_{i}, \operatorname{deg}\left(v_{i}\right)<N_{i}, \operatorname{deg}\left(d_{i}\right)<D_{i}$. We can apply the RFR componentwise and so, if $f_{i}=N_{i}+D_{i}-1$, we can uniquely reconstruct the solution.

Definition 2.1. (SRFR) Given $\boldsymbol{u}=\left(u_{1}, \ldots, u_{n}\right) \in \mathbb{K}[x]^{n}$ where $\operatorname{deg}\left(u_{i}\right)<f_{i}$, and degree bounds $0<N_{i}<f_{i}$ and $0<D<$ $\max _{1 \leq i \leq n}\left\{f_{i}\right\}$, we want to reconstruct the tuple $(\boldsymbol{v}, d)=\left(v_{1}, \ldots, v_{n}, d\right)$ such that

$$
v_{i} \equiv d u_{i} \bmod a_{i}, \operatorname{deg}\left(v_{i}\right)<N_{i}, \operatorname{deg}(d)<D .
$$

We denote $\mathcal{S}_{\boldsymbol{u}}$ the set of solutions.

The SRFR is then the problem of reconstructing a vector of rational functions with the same denominator. Therefore, if $f_{i}=$ $N_{i}+D-1$ for $1 \leq i \leq n$, we can uniquely reconstruct the solution. In this case, the common denominator property allows to reduce the number of unknowns, with an impact on the degree of the $a_{i}$ 's. In detail, the number of equations of (3) is $\sum_{i=1}^{n} f_{i}$, while the number of the unknowns, i.e. the coefficients of $v$ and $d$, is $\sum_{i=1}^{n} N_{i}+D$. If

$$
\sum_{i=1}^{n} f_{i}=\sum_{i=1}^{n} N_{i}+D-1
$$

then Equation (3) always admits a non-trivial solution. However, the uniqueness is not anymore guarantied.

Counterexample 2.2. Let $\mathbb{K}=\mathbb{F}_{11}, n=2, N_{1}=N_{2}=2, D=3$ and $a_{1}=a_{2}=\prod_{i=1}^{3}\left(x-2^{i}\right)=x^{3}+8 x^{2}+x+2$. Let $\boldsymbol{u}=\boldsymbol{v} / d$ with $\boldsymbol{v}=$
$(2 x+6,8 x+2)$ and $d=2 x^{2}+2 x+2$ invertible modulo $a_{i}$. Then the SRFR with instance $\boldsymbol{u}$ has two $\mathbb{K}[x]$-linearly independent solutions $(d, \boldsymbol{v})=\left(4 x^{2}+9 x+10,0,0\right)$ and $\left(d^{\prime}, \boldsymbol{v}^{\prime}\right)=(8 x+3,9 x+5,3 x+9)$.

Uniqueness is a central property for the applications of SRFR: unique decoding algorithms are essential in error correcting codes, and it is also a necessary condition to use evaluation interpolation techniques in computer algebra. The study of the bound on the number of equations which guaranties the uniqueness of SRFR has also repercussion on the complexity. Indeed, the complexity of decoding algorithms or evaluation interpolation techniques depends on this number of equations. So decreasing this number has a direct impact on the complexity.

We denote by $s$ the rank of the $\mathbb{K}[x]$-module spanned by the solutions $\mathcal{S}_{\boldsymbol{u}}$. Therefore, all solutions can be written as a linear combination $\sum_{i=1}^{s} c_{i} p_{i}$ of $s$ polynomials $p_{i}$ with polynomial coefficients $c_{i}$. The case $s=1$ corresponds to what we call uniqueness of the solution. In [OS07], the authors studied the particular case where $a_{1}=\ldots=a_{n}=a$ and $N_{1}=\ldots=N_{n}=N$. They proved the following,

TheOREM 2.3. [OS07, Theorem 4.2] Let $k$ be minimal such that $\operatorname{deg}(a) \geq N+(D-1) / k$, then the rank $s$ of the solution space $\mathcal{S}_{\boldsymbol{u}}$ satisfies $s \leq k$.

Note that if $k=1$, the solution is always unique $(s=1)$. This matches the uniqueness condition on the $\operatorname{deg}(a)$ of VRFR. On the other hand, if $k=n$ and $\operatorname{deg}(a) \geq N+(D-1) / n$ then $s \leq n$ which is always true. Hence in this case the theorem does not provide any new information about the solution space. This theorem represents a connection between the classic bound on the $\operatorname{deg}(a)=N+D-1$ which guaranties the uniqueness and the ideal one, i.e. $\operatorname{deg}(a)=$ $N+(D-1) / n$ (see Equation (4)), which exploits the common denominator property. They also proposed an algorithm that computes a complete basis of the solution space using $O\left(n k^{\omega-1} B(\operatorname{deg}(a))\right)$ operations in $\mathbb{K}$ where $2 \leq \omega \leq 3$ is the exponent of the matrix multiplication and $B(t):=M(t) \log t$ where $M$ is the classic polynomial multiplication arithmetic complexity (see [GG13] for instance). In [RS16] the complexity was improved. In particular, they introduced an algorithm that computes the solution space (in the general case of different moduli, i.e. $\left.a_{1}, \ldots, a_{n}\right)$ with complexity $O\left(n^{\omega-1} B(f) \log (f / n)^{2}\right)$ where $f=\max _{1 \leq i \leq n}\left\{\operatorname{deg}\left(a_{i}\right)\right\}$.

We now came back to general case of the SRFR. The main result of this work is to prove that when the degree constraints guarantee the existence of the solution, then for almost all $\boldsymbol{u}$ we also get the uniqueness (see Theorem 5.2).

THEOREM 2.4. If Equation (4) is satisfied, then for almost all instances $\boldsymbol{u}$ the SRFR admits a unique solution, i.e. it has rank $s=1$.

We will both use the expressions "almost all" or "generic", meaning that there exists a polynomial $R$ such that a certain property is true for all instances that do not cancel $R$. In our case, we state that there exists a polynomial $R$ such that the SRFR admits a unique solution for all instances $\boldsymbol{u}$ such that $R(\boldsymbol{u}) \neq 0$.

The SRFR problem has a natural application in a linear algebra context.

Application to polynomial linear system solving. Suppose that we want to compute the solution of a full rank polynomial linear system, $\boldsymbol{y}(x)=A^{-1} \boldsymbol{b} \in \mathbb{K}(x)$ where $A \in \mathbb{K}[x]^{n \times n}$ and $\boldsymbol{b} \in \mathbb{K}[x]^{n \times 1}$, 
from its image modulo a polynomial $a(x)$. We will refer to this problem as polynomial linear system solving (shortly PLS). We remark that, by the Cramer's rule, $\boldsymbol{y}$ is vector of rational functions with the same denominator: PLS is then a special case of SRFR. In [OS07], the authors proved that the solution space is uniquely generated $(s=1)$ when $\operatorname{deg}(a) \geq N+(D-1) / n$ in the special case of $D=N=n \operatorname{deg}(A)$ and $\operatorname{deg}(A)=\operatorname{deg}(b)$. They exploited another bound on the degree of $a$ based on [Cab71].

In view of Theorem 2.4 and as our experiments suggest, we could hope for the following,

CONJECTURe 2.5. If Equation (4) is satisfied then for almost all $(\boldsymbol{v}, d)$ with $\operatorname{gcd}\left(d, a_{i}\right)=1$, the SRFR with $\boldsymbol{u}=\frac{\boldsymbol{v}}{d}$ as input admits $a$ unique solution.

Since we have proved the uniqueness for generic instances $\boldsymbol{u}$, it would be sufficient to show the existence of an instance $\boldsymbol{u}$ of the form $v / d$ to prove the conjecture.

\subsection{Reconstruction with Errors}

In this section we introduce the problem of the Simultaneous Rational Function with Errors ([BK14, KPSW17, GLZ19, Per14, GLZ20]), i.e. the SRFR in a scenario where errors may occur in some evaluations. Throughout this section we suppose that $\mathbb{K}$ is a finite field of cardinality $q$, we fix $\boldsymbol{\alpha}=\left\{\alpha_{1}, \ldots, \alpha_{f}\right\}$ pairwise distinct evaluation points in $\mathbb{K}$ and we consider the polynomial $a=\prod_{i=1}^{f}\left(x-\alpha_{i}\right)$.

Definition 2.6. (SRFR with Errors) Fix $0<N, D, \varepsilon<f \leq q$. An instance of the SRFR with errors (SRFRwE) is a matrix $\omega \in \mathbb{K}^{n \times f}$ whose columns are $\boldsymbol{\omega}_{j}=\boldsymbol{v}\left(\alpha_{j}\right) / d\left(\alpha_{j}\right)+\boldsymbol{e}_{j}$ for some reduced $\boldsymbol{v} / d \in$ $\mathbb{K}(x)^{n \times 1}$ and some error matrix $\boldsymbol{e}$. The reduced vector must satisfy $\operatorname{deg}(\boldsymbol{v})<N, \operatorname{deg}(d)<D$ and $d\left(\alpha_{i}\right) \neq 0$. The error matrix must have its error support $E:=\left\{1 \leq j \leq f \mid \boldsymbol{e}_{j} \neq 0\right\}$ which satisfies $|E| \leq \varepsilon$.

The solution of the SRFRwE instance $\omega$ is $(\boldsymbol{v}, d)$.

SRFR wE as Reed-Solomon code decoding. We observe that if $n=1$ and $D=1, \boldsymbol{v} / d$ is a polynomial. Then the SRFRwE is the problem of recovering a polynomial $v$ given evaluations, some of which possibly erroneous. So in this case, SRFRwE is the problem of decoding an instance of a Reed-Solomon code.

Its vector generalization, that is $n>1$ and $D=1$, coincides with the decoding of an homogeneous Interleaved Reed-Solomon (IRS) code. Indeed, an IRS codeword can be seen as the evaluation of a vector of polynomials $\boldsymbol{v}$ on $\boldsymbol{\alpha}$. Thus decoding IRS codes is the problem of recovering $\boldsymbol{v}$ from $\omega_{j}=\boldsymbol{v}\left(\alpha_{j}\right)+\boldsymbol{e}_{j}$.

Let us now detail how we can solve SRFRwE using SRFR. We use the same technique of decoding RS and IRS codes [BW86, BKY03, PR17]. We introduce the Error Locator Polynomial $\Lambda=\prod_{j \in E}(x-$ $\left.\alpha_{j}\right)$. Its roots are the erroneous evaluations $\operatorname{so} \operatorname{deg}(\Lambda)=|E| \leq \varepsilon$. We consider the Lagrangian polynomials $u_{i} \in \mathbb{K}[x]$ such that $u_{i}\left(\alpha_{j}\right)=$ $\omega_{i j}$ for any $1 \leq i \leq n$. The classic approach is to remark that $(\boldsymbol{\varphi}, \psi)=(\Lambda(x) \boldsymbol{v}(x), \Lambda(x) d(x))$ is a solution of

$$
\boldsymbol{\varphi}=\psi \boldsymbol{u} \bmod \prod_{i=1}^{f}\left(x-\alpha_{i}\right) .
$$

In order to reconstruct $(\boldsymbol{v}, d)$ it suffices to study the set of $(\boldsymbol{\varphi}, \psi)$ which verify Equation (5) and such that $\operatorname{deg}(\varphi)<N+\varepsilon$ and $\operatorname{deg}(\psi)<$
$D+\varepsilon$. In this way we reduce SRFRwE to SRFR (see Eq. 3). Hence, if $f=(N+\varepsilon)+(D+\varepsilon)-1=N+D+2 \varepsilon-1$ we can uniquely reconstruct every component of the vector (cf. [BK14, KPSW17]).

It is possible to reduce the number of evaluations w.r.t. the maximal number of errors $\varepsilon$ in the setting of IRS decoding $(D=1)$.

Theorem 2.7 ([BKY03, BMS04, SSB09]). Fix $0<N, \varepsilon<f \leq q$ and $E$ such that $|E| \leq \varepsilon$. If $f=N-1+\varepsilon+\varepsilon / n$, then for all $(\boldsymbol{v}, 1)$ and almost all error matrices $\boldsymbol{e}$ of support E, the SRFRwE admits a unique solution on the instance $\omega$ where $\omega_{j}=\boldsymbol{v}\left(\alpha_{j}\right) / d\left(\alpha_{j}\right)+\boldsymbol{e}_{j}$.

We prove a similar result in the rational function case,

Theorem 2.8 ([GLZ19, GLZ20]). Fix $0<N, D, \varepsilon<f \leq q$ and E such that $|E| \leq \varepsilon$. If $f=N+D-1+\varepsilon+\varepsilon / n$, then for all $(\boldsymbol{v}, d)$ and almost all error matrices $\boldsymbol{e}$ of support $E$, the SRFRwE admits a unique solution on the instance $\omega$ where $\omega_{j}=\boldsymbol{v}\left(\alpha_{j}\right) / d\left(\alpha_{j}\right)+\boldsymbol{e}_{j}$.

Since the problem of SRFRwE reduces to a simultaneous rational function reconstruction, the Equation (5) always admits a nontrivial solution whenever $f=N+\varepsilon+(D+\varepsilon-1) / n$. Our ideal result would be to prove a uniqueness result also in this case. Our experiments suggest the following,

Conjecture 2.9. Fix $0<N, D, \varepsilon<f \leq q$ and $E$ such that $|E| \leq \varepsilon$. If $f=N+\varepsilon+(D+\varepsilon-1) / n$, then for almost all $(\boldsymbol{v}, d)$ and almost all error matrices $\boldsymbol{e}$ of support $E$, the SRFRwE admits a unique solution on the instance $\boldsymbol{\omega}$ where $\omega_{j}=\boldsymbol{v}\left(\alpha_{j}\right) / d\left(\alpha_{j}\right)+\boldsymbol{e}_{j}$.

Note that Conjecture 2.5 is for almost all fractions $(\boldsymbol{v}, d)$ whereas Theorems 2.7 and 2.8 are for all fractions. This difference is due to Counterexample 2.2, which states that we can not have uniqueness for all $(\boldsymbol{v}, d)$ when $f=N+(D-1) / n$. This latter number of evaluations matches the one of Conjecture 2.5 in the situation without errors $\varepsilon=0$. Remark that this obstruction does not affect Theorems 2.7 and 2.8 because their number of evaluations $f$ becomes $N+D-1$ when $\varepsilon=0$.

Our result Theorem 2.4 is a first step towards Conjecture 2.5: Since uniqueness of the SRFR is true generic instance $\omega_{j}$, it remains to prove the existence of an instance of the form $v\left(\alpha_{j}\right) / d\left(\alpha_{j}\right)+$ $\boldsymbol{e}_{j}$ for any $E$ such that $|E| \leq \varepsilon$ to prove the conjecture.

The SRFRwE was first introduced by [BK14] in a special case of its application, i.e. the Polynomial Linear System Solving with Errors, that we will introduce in the following paragraph.

Polynomial linear system solving with errors. We now suppose that we want to compute the unique solution of a PLS $y(x)=$ $\boldsymbol{v}(x) / d(x)=A^{-1} \boldsymbol{b} \in \mathbb{K}[x]^{n \times n}$ in a scenario where some errors occur [BK14, KPSW17, GLZ19]. In detail, we fix $f$ distinct evaluation points $\boldsymbol{\alpha}=\left\{\alpha_{1}, \ldots, \alpha_{f}\right\}$ such that $d\left(\alpha_{i}\right) \neq 0$. In our model, we suppose that there is a black box which for any evaluation point $\alpha_{i}$, gives a solution of the evaluated systems of linear equations, i.e. $\boldsymbol{y}_{i}=A\left(\alpha_{i}\right)^{-1} b\left(\alpha_{i}\right)$. However, this black box could do some errors in the computations. In particular, an evaluation $\alpha_{i}$ is erroneous if $\boldsymbol{y}_{i} \neq \boldsymbol{v}\left(\alpha_{i}\right) / d\left(\alpha_{i}\right)$ and we denote by $E:=\left\{i \mid \boldsymbol{y}_{i} \neq \boldsymbol{v}\left(\alpha_{i}\right) / d\left(\alpha_{i}\right)\right\}$ the set of erroneous positions. We refer to the problem of reconstructing the solution of a PLS in this model of errors as Polynomial Linear System Solving with Errors (shortly PLSwE). We observe that if $i \in E$, then there exists a nonzero $\boldsymbol{e}_{i} \in \mathbb{K}^{n \times f}$ such that $\boldsymbol{y}_{i}=\boldsymbol{v}\left(\alpha_{i}\right) / d\left(\alpha_{i}\right)+\boldsymbol{e}_{i}$. Hence, this problem is a special case 
of SRFRwE. Here we want to reconstruct a vector of rational functions which is a solution of a polynomial linear system. Therefore, all the results about uniqueness of the previous sections hold. Furthermore, in [KPSW17] authors introduced another bound which guaranties the uniqueness based on the bounds on the degree of the polynomial matrix $A$ and the vector $\boldsymbol{b}$.

\section{PRELIMINARIES}

In this section we will give some definitions and set out the notation that we will use throughout this paper. We refer to [Nei16] for the definitions and lemmas of this section, and for historical references.

\subsection{Row degrees of a $\mathbb{K}[x]$-module}

Let $\mathbb{K}$ be a field and $\mathbb{K}[x]$ the ring of polynomials over $\mathbb{K}$. We start by defining the row degree of a vector, then of a matrix. Let $\boldsymbol{p}=$ $\left(p_{1}, \ldots, p_{v}\right) \in \mathbb{K}[x]^{v}=\mathbb{K}[x]^{1 \times v}$ and $s=\left(s_{1}, \ldots, s_{v}\right) \in \mathbb{Z}^{v}$ a shift.

Definition 3.1 (Shifted row degree). Let $r_{i}=\operatorname{deg}\left(p_{i}\right)+s_{i}$ for $1 \leq$ $i \leq v$. The $s$-row degree of $\boldsymbol{p}$ is $\operatorname{rdeg}_{\boldsymbol{s}}(p)=\max _{1 \leq i \leq v}\left(r_{i}\right)$.

We also denote $\boldsymbol{p}=\left(\left[r_{1}\right]_{s_{1}}, \ldots,\left[r_{v}\right]_{s_{v}}\right)$ a vector of polynomials where $r_{i}=\operatorname{deg}\left(p_{i}\right)+s_{i}$.

We can extend this definition to polynomial matrices. In fact, let $P \in \mathbb{K}[x]^{\rho \times v}$ be a polynomial matrix, with $\rho \leq v$. Let $P_{j, *}$ be the $j$-th row of $P$ for $1 \leq j \leq \rho$. We can define the $s$-row degrees of the matrix $P$ as $\operatorname{rdeg}_{s}(P):=\left(r_{1}, \ldots, r_{\rho}\right)$ where $r_{j}:=\operatorname{rdeg}_{s}\left(P_{j, *}\right)$.

Let $\mathcal{N}$ be a $\mathbb{K}[x]$-submodule of $\mathbb{K}[x]^{v}=\mathbb{K}[x]^{1 \times v}$. Since $\mathbb{K}[x]$ is a principal ideal domain, $\mathcal{N}$ is free of $\operatorname{rank} \rho:=\operatorname{rank}(\mathcal{N})$ less than $v$ [DF03, Section 12.1, Theorem 4]. Hence, we can consider a basis $P \in \mathbb{K}[x]^{\rho \times v}$, i.e. a full rank polynomial matrix, such that $\mathcal{N}=\mathbb{K}[x]^{1 \times \rho} P=\left\{\lambda P \mid \lambda \in \mathbb{K}[x]^{1 \times \rho}\right\}$.

Our goal is to define a notion of row degrees of $\mathcal{N}$ in order to study later the $\mathbb{K}$-vector space $\mathcal{N}_{<r}:=\left\{\boldsymbol{p} \in \mathcal{N} \mid \operatorname{rdeg}_{\boldsymbol{s}}(\boldsymbol{p})<r\right\}$ for some $r \in \mathbb{N}$. Different bases $P$ of $\mathcal{N}$ have different $s$-row degrees so we need more definitions. We start with row reduced bases.

Let $\boldsymbol{t}=\left(t_{1}, \ldots, t_{v}\right) \in \mathbb{Z}^{v}$. We denote by $X^{\boldsymbol{t}}$ a diagonal matrix whose entries are $x^{t_{1}}, \ldots, x^{t_{v}}$.

Definition 3.2 (Shifted Leading Matrix). The $s$-leading matrix of $P$ is a matrix in $\mathbb{K}^{\rho \times v}$, whose entries are the coefficient of degree zero of $X^{-\operatorname{rdeg}_{s}(P)} P X^{s}$.

Definition 3.3. (Row reduced basis) A basis $P \in \mathbb{K}[x]^{\rho \times v}$ of $\mathcal{N}$ is $\boldsymbol{s}$-row reduced (shortly $\boldsymbol{s}$-reduced) if its leading matrix $L M_{\boldsymbol{s}}(P)$ has full rank.

This definition is equivalent to [Nei16, Definition 1.10], which implies that all $s$-reduced basis of $\mathcal{N}$ have the same row degree, up to permutation. We now focus on the following crucial property.

Proposition 3.4. (Predictable degree property)

$P$ is $\boldsymbol{s}$-reduced if and only if for all $\lambda=\left(\lambda_{1}, \ldots, \lambda_{\rho}\right) \in \mathbb{K}[x]^{1 \times \rho}$,

$$
r \operatorname{reg}_{s}(\lambda P)=\max _{1 \leq i \leq \rho}\left(\operatorname{deg}\left(\lambda_{i}\right)+r \operatorname{deg}_{s}\left(P_{i, *}\right)\right)=r \operatorname{deg}_{\boldsymbol{d}}(\lambda)
$$

where $\boldsymbol{d}=\operatorname{rdeg}_{\boldsymbol{s}}(P)$.

The proof of this classic proposition can be found for instance in [Nei16, Theorem 1.11]. This latter proposition is useful because it implies that $\operatorname{dim}_{\mathbb{K}} \mathcal{N}_{<r}=\sum_{\left\{i \mid r_{i}<r\right\}}\left(r-r_{i}\right)$ where $\left(r_{1}, \ldots, r_{\rho}\right)$ is the $s$-row degree of any $s$-reduced basis of $\mathcal{N}$.

Since we will need to define the $s$-row degrees of $\mathcal{N}$ uniquely, not just up to permutation, we need to introduce ordered weak Popov form, which relies on the notion of pivot.

Definition 3.5 (Pivot). Let $\boldsymbol{p} \in \mathbb{K}[x]^{1 \times v}$. The $\boldsymbol{s}$-pivot index of $\boldsymbol{p}$ is $\max \left\{j \mid \operatorname{rdeg}_{s}(\boldsymbol{p})=\operatorname{deg}\left(p_{j}\right)+s_{j}\right\}$. Moreover the corresponding $p_{j}$ is the $\boldsymbol{s}$-pivot entry and $\operatorname{deg}\left(p_{j}\right)$ is the $\boldsymbol{s}$-pivot degree of $\boldsymbol{p}$.

We can naturally extend the notion of pivot to polynomial matrices.

Definition 3.6. ((Ordered) weak Popov form) The basis $P$ of $\mathcal{N}$ in $s$-weak Popov form if the $s$-pivot indices of its rows are pairwise distinct. On the other hand, it is in s-ordered weak Popov form if the sequence of the $s$-pivot indices of its rows is strictly increasing.

A basis in $s$-weak Popov form is $s$-reduced. Indeed, $L M_{\boldsymbol{s}}(P)$ becomes, up to row permutation, a lower triangular matrix with nonzero entries on the diagonal. Hence it is full-rank.

Assume from now on that $\mathcal{N}$ is a submodule of $\mathbb{K}[x]^{v}$ of rank $v$ and that $P$ is a basis of $\mathcal{N}$ in $\boldsymbol{s}$-ordered weak Popov form. Then its pivot indices must be $\{1, \ldots, v\}$.

Weak Popov bases have a strong degree minimality property, stated in the following lemma.

Lemma 3.7 ([NeI16, Lemma 1.17]). Let $\boldsymbol{s} \in \mathbb{Z}^{v}, P$ be a basis of $\mathcal{N}$ in $\boldsymbol{s}$-weak Popov form with $\boldsymbol{s}$-pivot degrees $\left(d_{1}, \ldots, d_{v}\right)$. Let $\boldsymbol{p} \in \mathcal{N}$ whose pivot index is $1 \leq i \leq v$. Then the s-pivot degree of $\boldsymbol{p}$ is $\geq d_{i}$ or equivalently $r \operatorname{deg}_{s}(\boldsymbol{p}) \geq \operatorname{rdeg}_{\boldsymbol{s}}\left(P_{i, *}\right)$.

As it turns out, ordered weak Popov basis are reduced basis for which the $s$-row degree is unique. The following lemma is a consequence of Lemma 3.7.

Lemma 3.8 ([NeI16, Lemma 1.25]). Let $s \in \mathbb{Z}^{v}$ and assume $\mathcal{N}$ is a submodule of $\mathbb{K}[x]^{v}$ of rank $v$. Let $P$ and $Q$ be two bases of $\mathcal{N}$ in s-ordered weak Popov form. Then $P$ and $Q$ have the same s-row degrees and s-pivot degrees.

\subsection{Link between pivot and leading term}

In this section, we will focus on the relation between pivots of weak Popov bases and leading terms w.r.t. a specific monomial order, as in Gröbner basis theory (see for instance [CLO98]).

Let $\mathbb{K}[\boldsymbol{x}]:=\mathbb{K}\left[x_{1}, \ldots, x_{n}\right]$ be the ring of multivariate polynomials. Recall that a monomial in $\mathbb{K}[x]$ is a product of powers of the indeterminates $\boldsymbol{x}^{\boldsymbol{i}}:=x_{1}^{i_{1}} \cdots x_{n}^{i_{n}}$ for some $\boldsymbol{i}:=\left(i_{1}, \ldots, i_{n}\right) \in \mathbb{N}^{n}$. On the other hand, a monomial in $\mathbb{K}[x]^{n}$ is $\boldsymbol{x}^{\boldsymbol{i}} \varepsilon_{j}$, where $\varepsilon_{1}, \ldots, \varepsilon_{n}$ is the canonical basis of the $\mathbb{K}[x]$-module $\mathbb{K}[x]^{n}$.

A monomial order on $\mathbb{K}[x]^{n}$ is a total order $<$ on the monomials of $\mathbb{K}[\boldsymbol{x}]^{n}$ such that, for any monomials $\varphi \boldsymbol{\varepsilon}_{\boldsymbol{i}}, \psi \boldsymbol{\varepsilon}_{\boldsymbol{j}} \in \mathbb{K}[\boldsymbol{x}]^{n}$ and any monomial $\tau \neq 1, \tau \in \mathbb{K}[x]$,

$$
\varphi \varepsilon_{i} \prec \psi \varepsilon_{j} \Longrightarrow \varphi \varepsilon_{i} \prec \tau \varphi \varepsilon_{i} \prec \tau \psi \varepsilon_{j}
$$

Given a monomial order $<$ on $\mathbb{K}[\boldsymbol{x}]^{n}$ and $f \in \mathbb{K}[\boldsymbol{x}]^{n}$, the $<$-initial term in $<(f)$ of $f$ is the term of $f$ whose monomial is the greatest with respect to the order $\prec$. We remark that in the case of $\mathbb{K}[x]$, the only monomial order must be the natural degree order $x^{a}<$ $x^{b} \Longleftrightarrow a<b$. 
Definition 3.9. (shifted-TOP order) Let $\prec$ be a monomial order on $\mathbb{K}[\boldsymbol{x}]$. We consider the $\mathbb{K}[\boldsymbol{x}]$-module $\mathbb{K}[\boldsymbol{x}]^{n}$ with its canonical basis $\varepsilon_{1}, \ldots, \varepsilon_{n}$ and let $\gamma_{1}, \ldots, \gamma_{n}$ be monomials in $\mathbb{K}[x]$. Then $\prec$ induces the following monomial order on $\mathbb{K}[\boldsymbol{x}]^{n}$ called $s$-TOP (Term Over Position):

$$
\varphi \varepsilon_{i} \prec_{s-T O P} \psi \varepsilon_{j} \Longleftrightarrow\left(\varphi \gamma_{i} \prec \psi \gamma_{j}\right) \text { or }\left(\varphi \gamma_{i}=\psi \gamma_{j} \text { and } i<j\right)
$$

for any pairs of monomials $\varphi \varepsilon_{i}$ and $\psi \varepsilon_{j}$ of $\mathbb{K}[x]^{n}$.

As for the univariate module $\mathbb{K}[x]^{n}$, the only monomial order $\prec$ on $\mathbb{K}[x]$ is the natural one. The shifting monomials are $x^{s_{i}}$, defined by the shift $s=\left(s_{1}, \ldots, s_{n}\right) \in \mathbb{N}^{n}$. Hence, the $\boldsymbol{s}$-TOP order on $\mathbb{K}[x]^{n}$ is

$$
x^{a} \varepsilon_{i}<_{s \text {-TOP }} x^{b} \varepsilon_{j} \Longleftrightarrow\left(a+s_{i}, i\right)<_{\text {lex }}\left(b+s_{j}, j\right)
$$

where $<_{\text {lex }}$ is the lexicographic order on $\mathbb{Z}^{2}$.

We can now state the link between this monomial order and the pivot's definition: let $\boldsymbol{p} \in \mathbb{K}[x]^{1 \times n}$ and $i_{<_{s-\mathrm{TOP}}}(\boldsymbol{p})=\alpha x^{d} \boldsymbol{\varepsilon}_{i}$ be the $\prec_{s-T O P}$-initial term of $\boldsymbol{p}$, then the $s$-pivot index, entry, and degree are respectively $i, p_{i}$ and $d$. This will be useful later on, in e.g. Proposition 4.3 .

\section{ROW DEGREE OF THE RELATION MODULE}

Fix $m \geq n \geq 0$, and $M \in \mathbb{K}[x]^{m \times n}$. We consider a $\mathbb{K}[x]$-submodule $\mathcal{M}$ of $\mathbb{K}[x]^{n}$. We define the $\mathbb{K}[x]$-module homomorphism

$$
\begin{array}{rlc}
\hat{\varphi_{M}}: \mathbb{K}[x]^{m} & \longrightarrow \mathbb{K}[x]^{n} / \mathcal{M} \\
\boldsymbol{p} & \longmapsto & \boldsymbol{p} M
\end{array}
$$

Set $\mathcal{A}_{\mathcal{M}, M}:=\operatorname{ker}\left(\hat{\varphi_{M}}\right)$ to get the injection

$$
\varphi_{M}: \mathbb{K}[x]^{m} / \mathcal{A}_{\mathcal{M}, M} \hookrightarrow \mathbb{K}[x]^{n} / \mathcal{M} .
$$

We call $\mathcal{A}_{\mathcal{M}, M}$ the relation module because $p \in \mathcal{A}_{\mathcal{M}, M} \Leftrightarrow \varphi_{M}(\boldsymbol{p})=$ $p M=0 \bmod \mathcal{M}$, i.e. $p$ is a relation between rows of $M$.

Let $\varepsilon_{1}, \ldots, \varepsilon_{m}$ be the canonical basis of $\mathbb{K}[x]^{m}, \boldsymbol{\varepsilon}_{1}^{\prime}, \ldots, \boldsymbol{\varepsilon}_{n}^{\prime}$ the canonical basis of $\mathbb{K}[x]^{n}$ and $\boldsymbol{e}_{i} \equiv \varepsilon_{i} \bmod \mathbb{K}[x]^{m} / A_{\mathcal{M}, M}$ for $1 \leq$ $i \leq m$.

Remark 4.1. We observe that by the Invariant Factor Form of modules over Principal Ideal Domains (cf. [DF03, Theorem 4, Chapter $12]), \mathcal{K}:=\mathbb{K}[x]^{n} / \mathcal{M} \simeq \mathbb{K}[x]^{n} /\left\langle a_{i}(x) \varepsilon_{i}^{\prime}\right\rangle_{1 \leq i \leq n}$ for nonzeros $a_{i}(x) \in$ $\mathbb{K}[x]$ such that $a_{n}(x)\left|a_{n-1}(x)\right| \ldots \mid a_{1}(x)$. The polynomials $a_{i}(x)$ are the invariants of the module $\mathcal{M}$. We also denote $f_{i}:=\operatorname{deg}\left(a_{i}(x)\right)$ and we observe that $f_{1} \geq f_{2} \geq \ldots \geq f_{n}$.

From now on we will assume that $\mathcal{M}=\left\langle a_{i}(x) \varepsilon_{i}^{\prime}\right\rangle_{1 \leq i \leq n}$. It means that any $\boldsymbol{q} \in \mathcal{K}$ can be seen as $\left(q_{1} \bmod a_{1}, \ldots, q_{n} \bmod a_{n}\right)$. Using the result of Lemma 3.8, we can define the row and pivot degrees of the relation module $\mathcal{A}_{\mathcal{M}, M}$.

Definition 4.2 (Row and pivot degrees of the relation module). Let $s \in \mathbb{Z}^{m}$ be a shift and $P$ be any basis of $\mathcal{A}_{\mathcal{M}, M}$ in ordered weak Popov form. The $s$-row degrees of the relation module $\mathcal{A}_{\mathcal{M}, M}$ are $\boldsymbol{\rho}:=\operatorname{rdeg}_{\boldsymbol{s}}(P)=\left(\rho_{1}, \ldots, \rho_{m}\right)$ and the $\boldsymbol{s}$-pivot degrees are $\boldsymbol{\delta}:=$ $\left(\delta_{1}, \ldots, \delta_{m}\right)$ where $\delta_{i}=\rho_{i}-s_{i}$.

Throughout this paper we will also denote $\boldsymbol{\rho}_{M}$ and $\boldsymbol{\delta}_{M}$ when we want to stress out the matrix dependency.

\subsection{Row degree as row rank profile}

In this section, we will see that the row degrees of the relation module can be deduced from the row rank profile of a matrix associated to $\hat{\varphi}_{M}$. We start by associating the pivot degree of $\boldsymbol{p} \in \mathcal{A}_{\mathcal{M}, M}$ to linear dependency relation.

Proposition 4.3. There exists $\boldsymbol{p} \in \mathcal{A}_{\mathcal{M}, M}$ with $\boldsymbol{s}$-pivot index $i$ and $\boldsymbol{s}$-pivot degreed if and only if $x^{d} \boldsymbol{e}_{i} \in B_{M}^{<x^{d} \varepsilon_{i}}$ where $B_{M}^{<x^{d} \varepsilon_{i}}:=$ $\left\langle x^{n} \boldsymbol{e}_{j} \mid x^{n} \varepsilon_{j} \prec_{s-T O P} x^{d} \varepsilon_{i}\right\rangle$.

Proof. Fix $i, d \in \mathbb{N}$ and let $\boldsymbol{p} \in \mathbb{K}[x]^{n}$ with $s$-pivot index $i$ and $\boldsymbol{s}^{-}$ pivot degree $d$, so $r:=\operatorname{rdeg}_{s}(\boldsymbol{p})=d+s_{i}$. Then $\boldsymbol{p}=\left([\leq r]_{s_{1}}, \ldots,[\leq\right.$ $r]_{s_{i-1}},[r]_{s_{i}},[<r]_{s_{i+1}}, \ldots,[<r]_{s_{m}}$ ) (see Definition 3.1) and we can write $\boldsymbol{p}=c x^{d} \boldsymbol{\varepsilon}_{i}+\boldsymbol{p}^{\prime}$ where $c \in \mathbb{K}^{*}$ and $\boldsymbol{p}^{\prime}=\left([\leq r]_{s_{1}}, \ldots,[\leq\right.$ $\left.r]_{s_{i-1}},[<r]_{s_{i}},[<r]_{s_{i+1}}, \ldots,[<r]_{s_{m}}\right)$. So $\boldsymbol{p} \in \mathcal{A}_{\mathcal{M}, M}$ has $s$-pivot index $i$ and degree $d \Leftrightarrow x^{d} \varepsilon_{i}=-1 / c \boldsymbol{p}^{\prime} \bmod \mathcal{A}_{\mathcal{M}, M} \Leftrightarrow$

$x^{d} \boldsymbol{e}_{i} \in\left\langle x^{n} \boldsymbol{e}_{j} \mid \begin{array}{ll}n+s_{j} \leq d+s_{i}, & \text { for } 1 \leq j \leq i-1 \\ n+s_{j}<d+s_{i}, & \text { for } i \leq j \leq m\end{array}\right\rangle=B_{M}^{<x^{d} \varepsilon_{i}}$.

Theorem 4.4. Let $\boldsymbol{\delta}$ be the $\boldsymbol{s}$-pivot degrees of the relation module $\mathcal{A}_{\mathcal{M}, M}$. Then $\delta_{j}=\min \left\{d \mid x^{d} \boldsymbol{e}_{j} \in B_{M}^{<x^{d} \boldsymbol{\varepsilon}_{j}}\right\}$ for any $1 \leq j \leq m$.

Proof. Fix $1 \leq j \leq m$. During this proof we denote $\bar{\delta}_{j}:=$ $\min \left\{d \mid x^{d} \boldsymbol{e}_{j} \in B_{M}^{<x^{d} \varepsilon_{j}}\right\}$. We want to prove that $\delta_{j}=\bar{\delta}_{j}$. Recall that by Proposition 4.3, $x^{\delta_{j}} \boldsymbol{e}_{j} \in B_{M}^{<x^{\delta_{j}} \varepsilon_{j}}$. Hence, by the minimality of $\bar{\delta}_{j}, \delta_{j} \geq \bar{\delta}_{j}$. On the other hand, $x^{\bar{\delta}_{j}} \boldsymbol{e}_{j} \in B_{M}^{<x^{\bar{\delta}_{j}} \boldsymbol{\varepsilon}_{j}}$ so by Proposition 4.3 there exists $\boldsymbol{p} \in \mathcal{A}_{\mathcal{M}, M}$ of $\boldsymbol{s}$-pivot index $j$ and degree $\bar{\delta}_{j}$. Finally, by Lemma 3.7 we can conclude that $\bar{\delta}_{j} \geq \delta_{j}$.

We now define the ordered matrix $M o_{M}$ as the matrix of $\hat{\varphi}_{M}$ w.r.t. particular $\mathbb{K}$-vector space bases: the rows of $M o_{M}$ from top to bottom are the monomials of $\mathbb{K}[x]^{m}$ sorted increasingly for the $\prec_{s-T O P}$ order (see Eq. (6)). The columns of $M o_{M}$ are written w.r.t. the basis $\left\{x^{i} \varepsilon_{j}^{\prime}\right\}_{1 \leq j \leq n}$ of $\mathbb{K}[x]^{n} / \mathcal{M}$. Therefore, $M o_{M}$ has finite $0 \leq i<f_{j}$

$\operatorname{rank} \operatorname{rank}\left(M o_{M}\right)=\operatorname{rank}\left(\hat{\varphi}_{M}\right)=\operatorname{rank}\left(\varphi_{M}\right)$, infinite number of rows and $\left(\sum_{i=1}^{n} f_{i}\right)=\operatorname{dim}_{\mathbb{K}}\left(\mathbb{K}[x]^{n} / \mathcal{M}\right)$ columns.

Monomial row rank profile. Our goal is to relate the row rank profile of $M o_{M}$ to the row degree of the relation module. The classic definition of row rank profile of a rank $r$ polynomial matrix is the lexicographically smallest sequence of $r$ indices of linearly independent rows ( $c f$. [DPS15] for instance). Since the rows of our ordered matrix $\mathrm{Mo}_{M}$ correspond to monomials, we will transpose the previous definition to monomials instead of indices.

Let Mon $r$ be the sets of $r$ monomials of $\mathbb{K}[x]^{m}$. We define the lexicographical ordering on Mon $_{r}$ by comparing lexicographically the sorted monomials for $<_{s-T O P}$. In detail, $\mathcal{F}<_{\text {lex }} \mathcal{F}^{\prime}$ iff there exists $1 \leq t \leq r$ s.t. $x^{i_{l}} \varepsilon_{j_{l}}=x^{u_{l}} \varepsilon_{v_{l}}$ for $l<t$ and $x^{i_{t}} \varepsilon_{j_{t}}<_{s-T O P}$ $x^{u_{t}} \varepsilon_{v_{t}}$ where $\mathcal{F}=\left\{x^{i_{l}} \varepsilon_{j_{l}}\right\}_{1 \leq l \leq r}$ and $\mathcal{F}^{\prime}=\left\{x^{u_{l}} \varepsilon_{v_{l}}\right\}_{1 \leq l \leq r}$ and both $\left\{x^{i_{l}} \varepsilon_{j_{l}}\right\}$ and $\left\{x^{u_{l}} \varepsilon_{v_{l}}\right\}$ are increasing for the $<_{s-T O P}$ order.

We will use this lexicographic order on monomials to define the row rank profile of $M o_{M}$. Let $r=\operatorname{rank}\left(M o_{M}\right)$.

Definition 4.5 (Row rank profile). For any matrix $M \in \mathbb{K}[x]^{m \times n}$, we define the row rank profile of $M o_{M}$ (shortly $R R P_{M}$ ) as the family of monomials of $\mathbb{K}[x]^{m}$ defined by $R R P_{M}:=\min _{<_{\text {lex }}} \mathcal{P}_{M}$ where $\mathcal{P}_{M}:=\left\{\mathcal{F} \in \operatorname{Mon}_{r} \mid\{m M\}_{m \in \mathcal{F}}\right.$ are linearly independent in $\left.\mathcal{K}\right\}$. 
We now introduce a particular family of monomials, that we will frequently use: we will denote $\mathcal{F}_{\boldsymbol{d}}:=\left\{x^{i} \varepsilon_{j}\right\}_{i<d_{j}}$ for any $\boldsymbol{d}=\left(d_{1}, \ldots, d_{m}\right) \in \mathbb{N}^{m}$. $1 \leq j \leq m$

This family allows us to finally relate the row rank profile of $M o_{M}$ to the row degree of the relation module.

PROPOSITION 4.6. The row rank profile of the ordered matrix $\mathrm{Mo}_{M}$ is given by the pivot degrees $\boldsymbol{\delta}_{M}$ of the relation module $\mathcal{A}_{\mathcal{M}, M}$, i.e. $R R P_{M}=\mathcal{F}_{\delta_{M}}$.

Proof. We fix the matrix $M$ in order to simplify notations. We define $\delta_{j}^{\prime}=\min \left\{\delta \mid x^{\delta} \varepsilon_{j} \notin R R P\right\}$ and $\boldsymbol{\delta}^{\prime}=\left(\delta_{1}^{\prime}, \ldots, \delta_{m}^{\prime}\right)$. By properties of row rank profile, we have that $x^{\delta_{j}} \boldsymbol{e}_{j} \in B^{<x^{\delta_{j}}} \varepsilon_{j}$ (otherwise we could create a smaller family of linearly independent monomial with $x^{\delta_{j}} \boldsymbol{e}_{j}$ ). Using Theorem 4.4, we deduce that $\delta_{j}^{\prime} \geq \delta_{j}$. Therefore $\mathcal{F}_{\boldsymbol{\delta}} \subset \mathcal{F}_{\delta^{\prime}} \subset R R P$. Since the families of monomials $\mathcal{F}_{\boldsymbol{\delta}}$ and $R R P$ have the same cardinality $r=\operatorname{rank}(M o)$, they are equal so $\mathcal{F}_{\boldsymbol{\delta}}=R R P$.

\subsection{Constraints on relation's row degree}

We will now focus on integer tuples $\boldsymbol{\delta}_{M}$ which can be achieved. For this matter, in the light of Proposition 4.6, we need to understand which families $\mathcal{F}_{\boldsymbol{d}}$ of monomials can be linearly independent in the ordered matrix, i.e. belong to $\mathcal{P}_{M}$ (see Definition 4.5).

Recall that $\mathcal{K}=\mathbb{K}[x]^{n} / \mathcal{M}=\mathbb{K}[x]^{n} /\left\langle a_{i}(x) \varepsilon_{i}^{\prime}\right\rangle_{1 \leq i \leq n}$ and $f_{i}=$ $\operatorname{deg}\left(a_{i}(x)\right)$ are non-increasing as in Remark 4.1. Recall also from Definition 4.5 that $\mathcal{P}_{M}$ is the set of families $\mathcal{F}$ of $r$ monomials in $\mathbb{K}[x]^{m}$ such that $\{m M\}_{m \in \mathcal{F}}$ are linearly independent in $\mathbb{K}[x]^{n} / \mathcal{M}$.

Theorem 4.7. Let $\boldsymbol{d} \in \mathbb{N}^{m}$ be non-increasing. We can extend $\boldsymbol{f} \in$ $\mathbb{N}^{m}$ by $f_{n+1}=\ldots=f_{m}=0$. Then $\exists M \in \mathbb{K}[x]^{m \times n}$ such that $\mathcal{F}_{\boldsymbol{d}} \in \mathcal{P}_{M}$ if and only if $\sum_{i=1}^{l} d_{i} \leq \sum_{i=1}^{l} f_{i}$ for all $1 \leq l \leq m$.

The non-increasing property of $\boldsymbol{d}$ can be lifted: let $\boldsymbol{d}$ be nonincreasing and $\boldsymbol{d}^{\prime}$ be any permutation of $\boldsymbol{d}$. Then $\exists M \in \mathbb{K}[x]^{m \times n}$ such that $\mathcal{F}_{\boldsymbol{d}} \in \mathcal{P}_{M}$ if and only if $\exists M^{\prime} \in \mathbb{K}[x]^{m \times n}$ such that $\mathcal{F}_{\boldsymbol{d}^{\prime}} \in$ $\mathcal{P}_{M^{\prime}}$. Indeed, permuting $\boldsymbol{d}$ amounts to permuting the components of $p$,i.e. permuting the rows of $M$. This does not affect the existence property.

The latter proposition is an adaptation of [Vil97, Proposition 6.1] and its derivation [PS07, Theorem 3]. Even if the statements of these two papers are in a different but related context, their proof can be applied almost straightforwardly. We will still provide the main steps of the proof, for the sake of clarity and also because we will have to adapt the proof later in Theorem 5.2. Note also that we complete the 'if' part of the proof because it was not detailed in earlier references. For this matter, we introduce the following

Lemma 4.8. Let $\mathcal{N}$ be a $\mathbb{K}[x]$-submodule of $\mathcal{K}$ of rank $l$. Then the dimension of $\mathcal{N}$ as $\mathbb{K}$-vector space is at most $f_{1}+\ldots+f_{l}$.

Proof. First, remark that if $\boldsymbol{q} \in \mathcal{N}$ has its first non-zero element at index $p$ then $a_{p}(x) \boldsymbol{q}=0$. Now since $\mathcal{N}$ has rank $l$, we can consider the matrix $B$ whose rows are the $l$ elements of a basis of $\mathcal{N}$. We operate on the rows of $B$ to obtain the Hermite normal form $B^{\prime}$ of $B$ The rows $\left(\boldsymbol{b}_{i}^{\prime}\right)_{1 \leq i \leq l}$ of $B^{\prime}$ have first non-zero elements at distinct indices $k_{1}, \ldots, k_{l}$. Therefore $a_{k_{j}}(x) \boldsymbol{b}_{j}^{\prime}=0$ and $\left\{x^{i} \boldsymbol{b}_{j}^{\prime}\right\}_{0 \leq i<f_{k_{j}}}$ is a $1 \leq j \leq l$ generating set of $\mathcal{N}$ and so $\operatorname{dim}_{\mathbb{K}} \mathcal{N} \leq f_{k_{1}}+\ldots+f_{k_{l}} \leq f_{1}+\ldots+f_{l}$ since $\left(f_{i}\right)$ are non increasing and $\left(k_{j}\right)$ pairwise distinct.

Corollary 4.9. Let $r \geq 0, \boldsymbol{d} \in \mathbb{N}^{l}$ and $v_{1}, \ldots, v_{l} \in \mathcal{K}$ such that $\left\{x^{j} \boldsymbol{v}_{i}\right\}_{0 \leq j<d_{i}}$ are linearly independent then $\sum_{i=1}^{l} d_{i} \leq \sum_{i=1}^{l} f_{i}$. $1 \leq i \leq l$

Proof. We consider $\mathcal{N}$ the $\mathbb{K}[x]$-module spanned by $\left\{v_{1}, \ldots, v_{l}\right\}$, and we observe that $d_{1}+\ldots+d_{l} \leq \operatorname{dim} \mathcal{N} \leq f_{1}+\ldots+f_{l}$ by Lemma 4.8.

Proof of Theorem 4.7. We observe that if $m>n$, we can write $\mathcal{K}=\mathbb{K}[x]^{n} /\left\langle a_{i}(x) \varepsilon_{i}^{\prime}\right\rangle_{1 \leq i \leq n}=\mathbb{K}[x]^{m} /\left\langle a_{i}(x) \varepsilon_{i}\right\rangle_{1 \leq i \leq m}$ where $a_{j}(x)=$ 1 for $n+1 \leq j \leq m$. Hence we can suppose w.l.o.g. that $m=n$.

$\Rightarrow)$ By the hypotheses, there exists a matrix $M \in \mathbb{K}[x]^{m \times n}$ such that $\left\{x^{i} \varepsilon_{j} M\right\}_{x^{i} \varepsilon_{j} \in \mathcal{F}_{\boldsymbol{d}}}=\left\{x^{i} \boldsymbol{v}_{j}\right\}_{0<i<d_{j}}$ are linearly independent in $\mathcal{K}$ where $\boldsymbol{v}_{j}:=\varepsilon_{j} M$. Hence, for all $1 \leq l \leq m, \boldsymbol{v}_{1}, \ldots, \boldsymbol{v}_{l}$ satisfy the conditions of the Corollary 4.9 and so $\sum_{i=1}^{l} d_{i} \leq \sum_{i=1}^{l} f_{i}$.

$\Leftarrow)$ Set $\boldsymbol{u}_{i}=\varepsilon_{i}$ for $1 \leq i \leq m$ so that $\left\{x^{i} \boldsymbol{u}_{j}\right\}_{i<f_{j}}$ are linearly $1 \leq j \leq m$

independent in $\mathcal{M}$. We now consider the matrix $K:=\left[K_{1}|\ldots| K_{m}\right]$ where $K_{j} \in \mathbb{K}[x]^{m \times f_{j}}$ is in the Krylov form, that is $K_{j}=K\left(\boldsymbol{u}_{j}, f_{j}\right):=$ $\left[\boldsymbol{u}_{j}\left|x \boldsymbol{u}_{j}\right| \ldots \mid x^{f_{j}-1} \boldsymbol{u}_{j}\right]$ by considering $\boldsymbol{u}_{j}$ as a column vector. Note that $K$ is full column rank by construction. Our goal is to find vectors $\boldsymbol{v}_{1}, \ldots, \boldsymbol{v}_{m}$ such that $\left[K\left(\boldsymbol{v}_{1}, d_{1}\right)|\ldots| K\left(\boldsymbol{v}_{m}, d_{m}\right)\right]$ is full column $\operatorname{rank}$ (see $\widetilde{K}$ later).

For this matter, we first need to consider the matrix $\bar{K}$ made of columns of $K$ so that it remains full column rank. It is defined as $\bar{K}:=\left[\bar{K}_{1}|\ldots| \bar{K}_{m}\right]$ where for $1 \leq j \leq m, \bar{K}_{j} \in \mathbb{K}[x]^{m \times d_{j}}$ are defined iteratively by

$$
\bar{K}_{j}:=\left[K\left(\boldsymbol{u}_{j}, \min \left(f_{j}, d_{j}\right)\right)\left|K\left(x^{s_{1}} \boldsymbol{u}_{j_{1}}, t_{1}\right)\right| \ldots \mid K\left(x^{s_{k}} \boldsymbol{u}_{j_{k}}, t_{k}\right)\right]
$$

and $K\left(x^{s_{l}} \boldsymbol{u}_{j_{l}}, t_{l}\right)$ derives from previously unused columns in $K$, which we add from left to right, i.e. $\left(j_{l}\right)$ are increasing. Since $\sum_{i=1}^{j} d_{i} \leq$ $\sum_{i=1}^{j} f_{i}$, we will only pick from previous blocks, i.e. $j_{k}<j$. Since we must have depleted a block $K_{i_{l}}$ before going to another one, we can observe that $s_{l}+t_{l}=f_{l}$ for $l<k$. The last block $K_{i_{k}}$ is the only one that may not be exhausted, i.e. $s_{k}+t_{k} \leq f_{k}$. Conversely, $s_{l}=d_{l}$ for $l>1$ because no columns have been picked yet from the blocks $j_{l}$, except maybe the first block $j_{1}$ where $s_{1} \geq d_{1}$.

We want to transform $\bar{K}_{j}$ into a Krylov matrix $\widetilde{K}_{j}$, working block by block. First we extend $\left[K\left(\boldsymbol{u}_{j}, \min \left(f_{j}, d_{j}\right)\right)|0| \ldots \mid 0\right]$ to the right to $K\left(\boldsymbol{u}_{j}, d_{j}\right)$. Then we extend all blocks $\left[0|\ldots| 0\left|K\left(x^{s_{l}} \boldsymbol{u}_{j_{l}}, t_{l}\right)\right| 0|\ldots| 0\right]$ to the left and the right to $K\left(x^{s_{l}^{\prime}} \boldsymbol{u}_{j_{l}}, d_{l}\right)$ where $s_{l}^{\prime}$ equals $s_{l}$ minus the number of columns of the left extension. In this way, the extension matches the original matrix on its non-zero columns. Now we can define $\widetilde{K}:=\left[\widetilde{K}_{1}|\ldots| \widetilde{K}_{m}\right]$, where $\widetilde{K}_{j}:=K\left(\boldsymbol{v}_{j}, d_{j}\right)$ with $\boldsymbol{v}_{j}:=\boldsymbol{u}_{j}+$ $\sum_{l=1}^{k} x^{s_{l}^{\prime}} \boldsymbol{u}_{j_{l}}$.

A crucial point of the proof is to show that $s_{k}^{\prime} \geq 0$. But since $d_{i}$ are-non increasing, $j_{l}$ are increasing and $j_{k}<j$, we get $s_{l} \geq d_{j_{l}} \geq$ $d_{j_{k}} \geq d_{j}$. As the number of columns of the left extension is at most $d_{j}$, we can conclude $s_{k}^{\prime} \geq 0$.

In [Vil97] and [PS07] it is proved that there exist an upper triangular matrices $T$ such that $\widetilde{K}=\bar{K} T$. So we can conclude that $\widetilde{K}$, which is in the desired block Krylov form, is full column rank as is $\bar{K}$, which concludes the proof. 
Example 4.10. We illustrate the construction of the proof of Theorem 4.7 with example. Let $m=4, n=3, f=(8,4,4)$ extended to $f_{4}=0$ and $\boldsymbol{d}=(5,5,3,3)$. Remark that $\sum_{i=1}^{l} d_{i} \leq \sum_{i=1}^{l} f_{i}$ for all $1 \leq l \leq m$. Then $\bar{K}_{1}=K\left(\boldsymbol{u}_{1}, d_{1}\right), \bar{K}_{2}=\left[K\left(\boldsymbol{u}_{2}, f_{2}\right) \mid K\left(x^{d_{1}} \boldsymbol{u}_{1}, d_{2}-\right.\right.$ $\left.f_{2}\right)$ ] picks its missing column from the first unused column of $K_{1}$, $\bar{K}_{3}=K\left(\boldsymbol{u}_{3}, d_{3}\right)$, and $\bar{K}_{4}=\left[K\left(\boldsymbol{u}_{4}, f_{4}\right)=\varnothing \mid K\left(x^{d_{1}+1} \boldsymbol{u}_{1}, f_{1}-\left(d_{1}+\right.\right.\right.$ 1) $\left.\mid K\left(x^{d_{3}} \boldsymbol{u}_{3}, f_{3}-d_{3}\right)\right]$ picks its 3 missing columns first from the 2 unused of $K_{1}$, then from the remaining one of $K_{3}$. Then the construction extends $\bar{K}$ to $\widetilde{K}=K\left(\boldsymbol{v}_{i}, d_{i}\right)$ where $\boldsymbol{v}_{1}=\boldsymbol{u}_{1}=[1,0,0]$, $\boldsymbol{v}_{2}=\boldsymbol{u}_{2}+x^{d_{2}-\left(d_{1}-1\right)} \boldsymbol{u}_{1}=[x, 1,0], \boldsymbol{v}_{3}=\boldsymbol{u}_{3}=[0,0,1]$ and $\boldsymbol{v}_{4}=$ $x^{d_{1}+1} \boldsymbol{u}_{1}+x^{d_{3}-\left(f_{1}-\left(d_{1}+1\right)\right)} \boldsymbol{u}_{3}=\left[x^{6}, 0, x\right]$. Finally the matrix $M$ of the statement of Theorem 4.7 has its $j$-th row $M_{j, *}$ equal to $\boldsymbol{v}_{j}$. $\diamond$

We now have all the cards in our hand to state the principal constraint on the pivot degree $\boldsymbol{\delta}_{M}$ of the relation module $\mathcal{A}_{\mathcal{M}, M}$ when $M$ varies in the set of matrices $\mathbb{K}[x]^{m \times n}$ such that $\operatorname{rank}\left(M o_{M}\right)=$ $\operatorname{rank}\left(\varphi_{M}\right)$ is fixed. We will denote by $\mathbf{d}_{r}$ the pivot degree corresponding to the constraint.

THEOREM 4.11. Recall that $f=\left(f_{1}, \ldots, f_{m}\right)$ are the degrees of the invariants of $\mathcal{M}$ where $f_{i}=0$ for $n+1 \leq i \leq m$, and let $r=$ $\operatorname{rank}\left(M o_{M}\right)$. Then $\mathcal{F}_{\boldsymbol{\delta}_{M}} \geq_{\text {lex }} \mathcal{F}_{\mathbf{d}_{r}}$ where

$$
\mathcal{F}_{\mathbf{d}_{r}}=\min _{<_{l e x}}\left\{\mathcal{F}_{\boldsymbol{d}} \in \operatorname{Mon}_{r} \mid \forall 1 \leq l \leq m, \sum_{i=1}^{l} d_{i} \leq \sum_{i=1}^{l} f_{i}\right\}
$$

Proof. We know from Proposition 4.6 that $R R P_{M}=\mathcal{F}_{\boldsymbol{\delta}_{M}}$ so $\left\{x^{i} \varepsilon_{j} M\right\}_{i<\delta_{j, M}}$ are linearly independent and $\sum_{i=1}^{m} \delta_{i, M}=r$. Using $1 \leq j \leq m$

Theorem 4.7, we get that $\sum_{i=1}^{l} \delta_{i, M} \leq \sum_{i=1}^{l} f_{i}$ for all $1 \leq l \leq m$. This means that $\mathcal{F}_{\boldsymbol{\delta}_{M}}$ belongs to the set whose minimum is $\mathcal{F}_{\mathbf{d}_{r}}$, which implies our result.

We observe that $r=\operatorname{rank}\left(M o_{M}\right)$ must satisfy $0 \leq r \leq \Sigma:=$ $\sum_{i=1}^{m} f_{i}=\operatorname{dim}_{\mathbb{K}} \mathbb{K}[x]^{n} / \mathcal{M}$ and that $r=\sum$ is reachable since $m \geq n$. Note also that $\mathbf{d}_{r}$ is well-defined in Theorem 4.11 as long as $0 \leq$ $r \leq \Sigma:=\sum_{i=1}^{m} f_{i}$ because it is related to the minimum of a nonempty set.

\subsection{Generic row degree of relation module}

We will now show that this pivot degree constraint $\mathbf{d}_{\Sigma}$ is attainable by $\boldsymbol{\delta}_{M}$ for matrices $M$ such that $\operatorname{rank}\left(M o_{M}\right)=\operatorname{rank}\left(\varphi_{M}\right)=$ $\operatorname{dim}_{\mathbb{K}} \mathbb{K}[x]^{n} / \mathcal{M}$ in which case $\varphi_{M}$ becomes a bijection. More specifically, we will show that this is the case for almost all matrices $M \in \mathbb{K}[x]^{m \times n}$.

Corollary 4.12. For a generic matrix $M \in \mathbb{K}[x]^{m \times n}$, the pivot degrees $\boldsymbol{\delta}_{M}$ of the relation module $A_{\mathcal{M}, M}$ satisfy $\boldsymbol{\delta}_{M}=\mathbf{d}_{\Sigma}$ where $\Sigma=\sum_{i=1}^{n} f_{i}$.

Proof. Since $\sum_{i=1}^{l} \mathrm{~d}_{\Sigma, i} \leq \sum_{i=1}^{l} f_{i}$ for all $1 \leq l \leq m$, we deduce from Theorem 4.7 that there exists $M \in \mathbb{K}[x]^{m \times n}$ such that $\{m M\}_{m \in \mathcal{F}_{\mathbf{d}_{\Sigma}}}$ are linearly independent. So the $\Sigma$-minor corresponding to those lines is non-zero for this matrix $M$. We now consider this $\Sigma$-minor as a polynomial $R$ in the coefficients of $M$. This polynomial is then nonzero since it admits a nonzero evaluation.

Now for any matrix $M=\left(m_{i, j}\right)$ such that $R\left(m_{i, j}\right) \neq 0$, the vectors $\{m M\}_{m \in \mathcal{F}_{\mathbf{d}_{\Sigma}}}$ must be linearly independent, so $\operatorname{rank}\left(M o_{M}\right)=$ $\Sigma$. We have $R R P_{M} \leq_{\text {lex }} \mathcal{F}_{\mathbf{d}_{\Sigma}}$ because $\mathcal{F}_{\mathbf{d}_{\Sigma}} \in \mathcal{P}_{M}$ (see Definition 4.5).
Theorem 4.11 gives the other inequality, so $\mathcal{F}_{\mathbf{d}_{\Sigma}}=R R P_{M}=\mathcal{F}_{\boldsymbol{\delta}_{M}}$ and $\boldsymbol{\delta}_{M}=\mathbf{d}_{\Sigma}$.

4.3.1 Special cases. In this section, we will see that our definition of the generic pivot degree $\mathbf{d}_{\Sigma}$ in Eq. (7) has a simplified expression in a wide range of settings. Set the notation $\bar{s}=\max (s)$. We will see that under some assumptions the expected row degree $\mathbf{p}_{\Sigma}:=\mathbf{d}_{\Sigma}+\boldsymbol{s}$ has a nice form. Define $p$ and $u$ be the quotient and remainder of the Euclidean division $\sum_{i=1}^{m}\left(f_{i}+s_{i}\right)=p \cdot m+u$. The expected nice form of the row degrees will be

$$
\boldsymbol{p}:=(\underbrace{p+1, \ldots, p+1}_{u \text { times }}, \underbrace{p, \ldots, p}_{m-u \text { times }}) .
$$

This nice form will appear the following conditions on $f$ and $s$ :

$$
\begin{gathered}
p \geq \bar{s} \\
\forall 1 \leq l \leq m-1, \sum_{i=1}^{l} p_{i} \leq \sum_{i=1}^{l}\left(f_{i}+s_{i}\right)
\end{gathered}
$$

THEOREM 4.13. Letp as in Equation (8), ant let $f$ be non-increasing such that Equations (9) and (10) hold. Then $\mathbf{p}_{\Sigma}=\boldsymbol{p}$.

This nice form of row degree was already observed in particular cases in different but related settings. To the best of our knowledge, it can be found in [Vil97, Proposition 6.1] for row degrees of minimal generating matrix polynomial but with no shift, in [PS07, Corollary 1] for dimensions of blocks in a shifted Hessenberg form but the link to row degree is unclear and no shift is discussed (shifted Hessenberg is not related to our shift $s$ ), and in [JV05, after Eq. (2)] for kernel basis were $m=2 n$ with no shifts.

Proof. Denote again $\Sigma=\sum_{i=1}^{n} f_{i}$. Let $\overline{\mathcal{F}}$ be the first $\Sigma$ monomials of $\mathbb{K}[x]^{m}$ for the $<_{s-T O P}$ ordering. Let $\boldsymbol{p}=(p+1, \ldots, p+$ $1, p, \ldots, p)$ be the candidate row degrees as in the theorem statement and $\boldsymbol{d}=\boldsymbol{p}-\boldsymbol{s}$ be the corresponding pivot degrees. Note that Equation (9) implies that $p \geq \bar{s}$ so $\boldsymbol{d} \in \mathbb{N}^{m}$.

First we show that Equation (9) implies $\overline{\mathcal{F}}=\mathcal{F}_{\boldsymbol{d}}$. For the first part, in order to prove $\overline{\mathcal{F}}=\mathcal{F}_{\boldsymbol{d}}$, we need to show that $d_{i}=\min \{d \in$ $\left.\mathbb{N} \mid x^{d} \varepsilon_{i} \notin \overline{\mathcal{F}}\right\}$. We already know that $d_{i} \in \mathbb{N}$. We will need to study the row degrees of the first monomials to conclude. The monomials of $\mathbb{K}[x]^{m}$ of $s$-row degree $r$ ordered increasingly for $<_{s-T O P}$ are $\left[x^{r-s_{i}} \varepsilon_{i}\right]$ for increasing $1 \leq i \leq m$ such that $s_{i} \leq r$. There are $m$ such monomials when $r \geq \bar{s}$. The monomials of $s$-row degree less than $\bar{s}$ are $\left\{x^{i} \varepsilon_{j}\right\}_{i+s_{j}<\bar{s}}$ and their number is $\sum_{i=1}^{m}\left(\bar{s}-s_{i}\right)$. From this we can deduce that the row degree of the $n$-th smallest monomial is $\left\lfloor\left(n-1-\sum_{i=1}^{m}\left(\bar{s}-s_{i}\right)\right) / m\right\rfloor+\bar{s}=\left\lfloor\left(n-1+\sum_{i=1}^{m} s_{i}\right) / m\right\rfloor$ provided that $n \geq \sum_{i=1}^{m}\left(\bar{s}-s_{i}\right)+1$. We can now remark that the $(\Sigma+1)$-th smallest monomial has $s$-row degree $p$. More precisely, the $(\Sigma+1)$ th smallest monomial is the $(u+1)$-th monomial of row-degree $r$, so $\overline{\mathcal{F}}$ is equal to all monomials of row degree less than $p$ and the first $u$ monomials of row degree $p$. This proves $d_{i}=\min \left\{d \in \mathbb{N} \mid x^{d} \varepsilon_{i} \notin\right.$ $\overline{\mathcal{F}}\}$ and $\overline{\mathcal{F}}=\mathcal{F}_{\boldsymbol{d}}$.

Second we deduce from Equation (10) that for all $1 \leq l \leq m$, $\sum_{i=1}^{l} d_{i}=\sum_{i=1}^{l}\left(p_{i}-s_{i}\right) \leq \sum_{i=1}^{l} f_{i}$, so $\mathcal{F}_{\mathbf{d}_{r}} \leq_{\text {lex }} \mathcal{F}_{\boldsymbol{d}}$ by Theorem 4.11 and finally $\mathcal{F}_{\mathbf{d}_{r}}=\mathcal{F}_{\boldsymbol{d}}$ because $\overline{\mathcal{F}}$ is the smallest set of $\Sigma$ monomials. 
Example 4.14. Here we provide 3 examples of generic row pivot $\mathbf{d}_{\Sigma}$ and row degree $\mathbf{p}_{\Sigma}$ : Corollary 4.12 applies only to the first situation because the second and third situations are made so that Eq. (9) and respectively Eq. (10) are not satisfied. Let $m=n=3$ and $s=(0,2,4)$ so that $\bar{s}=4$ and $\sum\left(\bar{s}-s_{i}\right)=6$.

In the first situation $f=(6,1,0)$, so $\sum\left(f_{i}+s_{i}\right)=4 * m+1$ and using Corollary 4.12 we get $\mathbf{p}_{\Sigma}=(5,4,4)$ from Eq. (8) and $\mathbf{d}_{\Sigma}=(5,2,0)$. In the second situation, $f=(3,0,0)$ and Eq. (9) is not satisfied. We use Theorem 4.13 to get $\mathbf{d}_{\Sigma}=(3,0,0)$ from Eq. (7) and $\mathbf{p}_{\Sigma}=(3,2,4)$. Finally in the third situation, $f=(3,3,1)$ and Eq. (10) is not satisfied. We use Theorem 4.13 to get $\mathbf{d}_{\Sigma}=(3,3,1)$ from Eq. (7) and $\mathbf{p}_{\Sigma}=(3,5,5)$. Let $\mathcal{F}_{1}, \mathcal{F}_{2}, \mathcal{F}_{3}$ be the respective families of monomial of the three situations. We picture these families in the following table, where Mon are the first monomials for $<_{s-T O P}$

\begin{tabular}{r|ccc|ccccccc}
\hline$M o n$ & $\varepsilon_{1}$ & $X \varepsilon_{1}$ & $X^{2} \varepsilon_{1}$ & $\varepsilon_{2}$ & $X^{3} \varepsilon_{1}$ & $X \varepsilon_{2}$ & $X^{4} \varepsilon_{1}$ & $X^{2} \varepsilon_{2}$ & $\varepsilon_{3}$ \\
\hline $\operatorname{rdeg}_{s}$ & 0 & 1 & & 2 & & 3 & & 4 & \\
\hline \hline $\mathcal{F}_{1}$ & $\bullet$ & $\bullet$ & $\bullet$ & $\bullet$ & $\bullet$ & $\bullet$ & $\bullet$ & & \\
\hline $\mathcal{F}_{2}$ & $\bullet$ & $\bullet$ & $\bullet$ & & & & & & \\
\hline $\mathcal{F}_{3}$ & $\bullet$ & $\bullet$ & $\bullet$ & $\bullet$ & & $\bullet$ & & $\bullet$ & $\bullet$ \\
\hline
\end{tabular}

\section{UNIQUENESS RESULTS ON SRFR}

Recall the SRFR, defined in Section 2.1. In particular, $a_{1}, \ldots, a_{n} \in$ $\mathbb{K}[x]$ with degrees $f_{i}:=\operatorname{deg}\left(a_{i}\right)$ and $\boldsymbol{u}:=\left(u_{1}, \ldots, u_{n}\right) \in \mathbb{K}[x]^{n}$ such that $\operatorname{deg}\left(u_{i}\right)<f_{i}$ and $0<N_{i} \leq f_{i}$ for $1 \leq i \leq n, 0<D \leq$ $\min _{1 \leq i \leq n}\left\{f_{i}\right\}$. We want to reconstruct $(\boldsymbol{v}, d)=\left(v_{1}, \ldots, v_{n}, d\right) \in$ $\mathbb{K}[x]^{1 \times(n+1)}$ such that $v_{i} \equiv d u_{i} \bmod a_{i}, \operatorname{deg}\left(v_{i}\right)<N_{i}, \operatorname{deg}(d)<D$.

We consider $\mathcal{M}=\left\langle a_{i}(x) \varepsilon_{i}^{\prime}\right\rangle$ and we denote by $S_{\boldsymbol{u}}$ the set of tuples which verify Eq. (3).

LEMmA 5.1. For the shift $s=\left(-N_{1}, \ldots,-N_{n},-D\right) \in \mathbb{Z}^{n+1}$, we have $(\boldsymbol{v}, d) \in S_{\boldsymbol{u}} \Leftrightarrow(\boldsymbol{v}, d) \in \mathcal{A}_{\mathcal{M}, R_{\boldsymbol{u}}}$ with $\operatorname{rdeg}_{\boldsymbol{s}}((\boldsymbol{v}, d))<0$, where

$$
R_{\boldsymbol{u}}:=\left[\begin{array}{c}
\operatorname{Id}_{n} \\
-\boldsymbol{u}
\end{array}\right] \in \mathbb{K}[x]^{(n+1) \times n}
$$

Proof. Observe that $(\boldsymbol{v}, d) \in S_{\boldsymbol{u}}$ if and only if it satisfies the equation $\boldsymbol{v}-d \boldsymbol{u} \equiv(\boldsymbol{v}, d) R_{\boldsymbol{u}} \equiv 0 \bmod \mathcal{M}$, that is $(\boldsymbol{v}, d) \in \mathcal{A}_{\mathcal{M}, R_{\boldsymbol{u}}}$, and if it satisfies the degree conditions equivalent to $\operatorname{rdeg}_{s}((\boldsymbol{v}, d))=$ $\max \left\{\operatorname{deg}\left(v_{1}\right)-N_{1}, \ldots, \operatorname{deg}\left(v_{n}\right)-N_{n}, \operatorname{deg}(d)-D\right\}<0$ (see Definition 3.1).

So in order to study the solutions of the SRFR we introduce the $\boldsymbol{s}$-row degrees $\boldsymbol{\rho}_{\boldsymbol{u}}:=\boldsymbol{\rho}_{R_{\boldsymbol{u}}}$ and the $\boldsymbol{s}$-pivot indices $\boldsymbol{\delta}_{\boldsymbol{u}}:=\boldsymbol{\delta}_{R_{\boldsymbol{u}}}$ of $A_{R_{\boldsymbol{u}}, \mathcal{M}}$ (see Definition 4.2). As remarked just after the predictable degree property (Proposition 3.4),

$$
\operatorname{dim}_{\mathbb{K}} S_{\boldsymbol{u}}=\operatorname{dim}_{\mathbb{K}}\left(A_{R_{\boldsymbol{u}}, \mathcal{M}}\right)_{<0}=-\sum_{\rho_{\boldsymbol{u}, i}<0} \rho_{\boldsymbol{u}, i} .
$$

We can now show our main theorem about uniqueness in SRFR for generic instances $\boldsymbol{u}$.

Theorem 5.2. Assume $\sum_{i=1}^{n} f_{i}=\sum_{i=1}^{n} N_{i}+D-1$. Then for generic $\boldsymbol{u}=\left(u_{1}, \ldots, u_{n}\right) \in \mathbb{K}[x]^{1 \times n}$, the solution space $S_{\boldsymbol{u}}$ has dimension 1 as $\mathbb{K}$-vector space.

Proof. By the previous considerations (see Eq. (12)) it is sufficient to prove that for generic $\boldsymbol{u} \in \mathbb{K}[x]^{n+1}, \boldsymbol{\rho}_{\boldsymbol{u}}=(0, \ldots, 0,-1)$.

First, we need to show that the generic $\boldsymbol{s}$-row degree $\mathbf{p}_{\Sigma}$ is the expected nice form $p=(0, \ldots, 0,-1)(p=-1$ and $u=n=m-1$ because $\sum\left(f_{j}+s_{j}\right)=-1 \cdot m+(m-1)$, see Eq. (8)). It remains to check that we verify the hypotheses of Theorem 4.13. By Equation (9), $\bar{s} \leq-1=p$. By Equation (10), $\sum_{i=1}^{l} p_{i} \leq 0 \leq \sum_{i=1}^{l}\left(f_{i}+s_{i}\right)$ for all $0 \leq l \leq m-1$ since $f_{i}+s_{i} \geq 0 \geq p_{i}$ for all $i$.

It remains to show that there exists a matrix of the form $R_{\boldsymbol{u}}$ which satisfies the genericity condition of Corollary 4.12. Hence, the genericity condition is a non-zero polynomial when evaluated on matrices $R_{\boldsymbol{u}}$ and finally we have our result for generic $\boldsymbol{u}$.

In order to do so, we show that the construction of the proof of the Theorem 4.7 provides a matrix of the form $R_{\boldsymbol{u}}$ in our case. In our case $\left(d_{1}, \ldots, d_{n+1}\right)=\left(N_{1}, \ldots, N_{n}, D-1\right)$ and $m=n+1$, where $f_{n+1}=0$. In particular, by SRFR assumptions, for any $1 \leq i \leq n$, $d_{i} \leq f_{i}$ and so the matrices $\bar{K}_{i}=\left[K\left(\boldsymbol{u}_{i}, d_{i}\right)\right]$ are already in the Krylov form. On the other hand, the last matrix is in the form $\bar{K}_{n+1}=\left[K\left(x^{d_{j}} \boldsymbol{u}_{j}, t_{j}\right)\right]_{1 \leq j \leq n}$ where $d_{j}+t_{j}=f_{j}$. Then $\widetilde{K}_{n+1}=$ $\left[K\left(\sum_{j=1}^{n} x^{s_{j}^{\prime}} \boldsymbol{u}_{j}, d_{j}\right)\right]$ and we need to prove that $s_{j}^{\prime} \geq 0$ differently because we don't have the assumption about the non-increasing $\boldsymbol{d}$. Recall that $s_{j}^{\prime}$ is $s_{j}$ minus the number of columns added to extend the matrix to the left. This number of columns is at most $d_{n+1}$ minus the size $t_{l}$ of the current block. So $s_{l}^{\prime} \geq d_{l}-\left(d_{n+1}-t_{l}\right)=$ $d_{l}-\left(d_{n+1}-\left(f_{l}-d_{l}\right)\right)=f_{l}-d_{n+1} \geq 0$ because $d_{n+1}=D-1 \leq$ $D \leq \min \left(f_{i}\right)$ and so the construction works.

\section{REFERENCES}

[BK14] B. Boyer and E. Kaltofen. Numerical linear system solving with parametric entries by error correction. In Proceedings of SNC'14, pages 33-38, New York, NY, USA, 2014. ACM.

[BKY03] D. Bleichenbacher, A. Kiayias, and M. Yung. Decoding of interleaved reed solomon codes over noisy data. In Proceedings of ICALP'03, pages 97-108, 2003.

[BMS04] A. Brown, L. Minder, and A. Shokrollahi. Probabilistic decoding of interleaved RS-codes on the q-ary symmetric channel. In Proceedings of ISIT'04, pages 326-326, 2004

[BW86] E. R. Berlekamp and L. R. Welch. Error correction of algebraic block codes., 1986.

[Cab71] S. Cabay. Exact solution of linear equations. In Proceedings of SYMSAC'71, pages 392-398, New York, NY, USA, 1971. Association for Computing Machinery.

[CLO98] D. Cox, J. Little, and D. O'Shea. Using algebraic geometry, volume 185 of Graduate Texts in Mathematics. Springer-Verlag, New York, 1998.

[DF03] D. S. Dummit and R. M. Foote. Abstract Algebra. Wiley, 3nd. edition, 2003.

[DPS15] J.-G. Dumas, C. Pernet, and Z. Sultan. Computing the Rank Profile Matrix. In Proceedings of ISSAC'15, pages 149-156, New York, NY, USA, 2015. ACM.

[GG13] J. von zur Gathen and J. Gerhard. Modern Computer Algebra. Cambridge University Press, 3 edition, 2013.

[GLZ19] E. Guerrini, R. Lebreton, and I. Zappatore. Polynomial linear system solving with errors by simultaneous polynomial reconstruction of interleaved reed-solomon codes. In Proceedings of ISIT'19, pages 1542-1546, 2019.

[GLZ20] E. Guerrini, R. Lebreton, and I. Zappatore. Enhancing simultaneous rational function recovery: adaptive error correction capability and new bounds for applications. Submitted, 2020.

[JV05] C.-P. Jeannerod and G. Villard. Essentially optimal computation of the inverse of generic polynomial matrices. Fournal of Complexity, 21(1):7286, 2005.

[KPSW17] E. L. Kaltofen, C. Pernet, A. Storjohann, and C. Waddell. Early termination in parametric linear system solving and rational function vector recovery with error correction. In Proceedings of ISSAC'17, pages 237-244, New York, NY, USA, 2017. ACM.

[Nei16] V. Neiger. Bases of relations in one or several variables: fast algorithms and applications. phdthesis, ÃL'cole Normale SupÃ lrieure de Lyon - University of Waterloo, 2016.

[OS07] Z. Olesh and A. Storjohann. The vector rational function reconstruction problem. In Proceedings of the Waterloo Workshop, pages 137-149. World Scientific, 2007.

[Per14] C. Pernet. High Performance and Reliable Algebraic Computing. Habilitation à diriger des recherches, Université Joseph Fourier, Grenoble 1, 2014. 
[PR17] S. Puchinger and J. Rosenkilde né Nielsen. Decoding of interleaved reedsolomon codes using improved power decoding. In Proceedings of ISIT'17, pages 356-60. IEEE, 2017.

[PS07] C. Pernet and A. Storjohann. Faster Algorithms for the Characteristic Polynomial. In Proceedings of ISSAC'07, pages 307-314, New York, NY, USA, 2007. ACM. event-place: Waterloo, Ontario, Canada.

[RS16] J. Rosenkilde né Nielsen and A. Storjohann. Algorithms for simultaneous padé approximations. In Proceedings of ISSAC'16, page 405âĂŞ412, New
York, NY, USA, 2016. Association for Computing Machinery.

[SSB09] G. Schmidt, V. R. Sidorenko, and M. Bossert. Collaborative decoding of interleaved reedâĂŞsolomon codes and concatenated code designs. IEEE Transactions on Information Theory, 55(7):2991-3012, 2009.

[Vil97] G. Villard. A study of Coppersmith's block Wiedemann algorithm using matrix polynomials. IMAG, Institut d'informatique et de mathÃlmatiques appliquÃles de Grenoble, 1997. 